\title{
Déficit de vitamina D en pacientes en hemodiálisis y factores relacionados
}

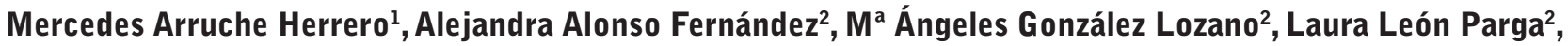 \\ Rosa Ana Paz Paz², Edith García Abello², Rocío Martos Bustos², Dolors Sabaté Rovira², Carmen Palomares \\ Peña², Javier Varas García ${ }^{3}$ \\ ${ }^{1}$ Nefróloga, Fresenius Medical Care. Reus. Tarragona. España \\ 2 D.U.E, Fresenius Medical Care. Reus. Tarragona. España \\ ${ }^{3}$ Doctor en Biología,Departamento Médico, Fresenius Medical Care. Madrid. España
}

\section{Resumen}

Introducción: La vitamina $D$ interviene en el metabolismo óseo-mineral, la diabetes mellitus, enfermedades inmunológicas, procesos tumorales, el sistema cardiovascular, anemia, etc.

Objetivos: Determinar la prevalencia de insuficiencia-deficiencia de vitamina $D$ y los factores de riesgo asociados en pacientes en hemodiálisis en Reus.

Material y Método: Realizamos en abril de 2017 la determinación de los niveles sanguíneos, una entrevista oral sobre hábitos estables de adquisición de vitamina D y se extrajeron de la historia clínica enfermedades relacionadas, tratamientos e IMC.

Resultados: Solo el 5,88\% de los pacientes presentaban niveles normales. El 94,14\% presentaron niveles inferiores a $30 \mathrm{ng} / \mathrm{ml}$ (promedio 9,64 ng/ml). En este grupo los pacientes resultaron de mayor edad, se exponen menos al sol, utilizan protección solar, hay mayor presencia de mujeres, toman menos alimentos enriquecidos o ricos en vitamina $D$ y menos estatinas. Entre ellos se encuentran los tres pacientes con peso insuficiente. Los dos pacientes de raza negra tenían niveles $<10 \mathrm{ng} / \mathrm{ml}$. En nuestra situación geográfica, Latitud: $41^{\circ} 09^{\prime} 22^{\prime \prime} \mathrm{N}$, en invierno la fotobiosíntesis de vitamina D es escasa. Solo el $14,12 \%$ de los pacientes tienen

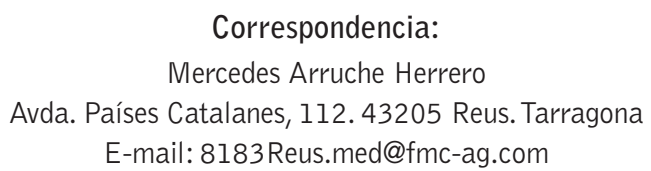

prescrito alguna suplementación de vitamina D. Sólo una variable fue estadísticamente significativa (raza) lo que puede atribuirse al tamaño limitado de la muestra.

Conclusiones: El 94,14\% de los pacientes tienen niveles bajos vitamina $D$ y parece necesaria su suplementación farmacológica si queremos normalizarlos.

PALABRAS CLAVE: vitamina D; hemodiálisis; prevalencia; factores de riesgo.

\section{Deficit of vitamin D in hemodialysis patients and related factors}

\section{Abstract}

Introduction: Vitamin $D$ is involved in bone-mineral metabolism, diabetes mellitus, immunological diseases, tumor processes, cardiovascular system, anemia, etc.

Aims: To evaluate the prevalence of vitamin D insufficiency- deficiency and its associated risk factors in hemodialysis patients.

Results: Only $5.88 \%$ of patients presented normal levels. $94.14 \%$ of patients had levels below $30 \mathrm{ng} / \mathrm{ml}$ (mean $9.64 \mathrm{ng} / \mathrm{ml}$ ). In this last group, patients were older, less exposed to the sun and used sun protection. A greater presence of women was found, taking less vitamin $D$ or VD enriched foods and fewer statins. Between them, three patients had insufficient weight. The two black patients had levels $<10 \mathrm{ng} / \mathrm{ml}$. In our geographical situation [Latitude: $41^{\circ} 09^{\prime} 22^{\prime \prime} \mathrm{N}$ ], in winter, vitamin D skin-synthesis is scarce. Only $14.12 \%$ of patients took 
any vitamin D supplementation. Only one variable was statistically significant (human race). We must consider that it can be attributed to our limited sample size.

Conclusions: $94.14 \%$ of patients have low vitamin D levels and pharmacological supplementation seems necessary.

KEYWORDS: vitamin D; hemodialysis; prevalence; Risk factors.

\section{Introducción}

Es conocida la importancia de la vitamina $D$ (VD) en el metabolismo óseo-mineral, así como su asociación establecida en los últimos años con la diabetes mellitus (DM), enfermedades inmunológicas, procesos tumorales, el sistema cardiovascular, anemia, mortalidad, etc ${ }^{1,2}$. También se conoce que su déficit o insuficiencia acontece en todo el planeta variando su prevalencia según los grupos estudiados 3,4 .

La VD se forma a partir de 7-dehidrocolesterol en la piel por irradiación UVB. En el hígado la VD se hidroxila para formar 25-(OH)D (calcidiol), que se metaboliza a su metabolito activo 1,25(OH)2D (calcitriol), por la enzima CYP27B1, preferentemente en el riñón. La vitamina $\mathrm{D}$ también puede ser aportada por la dieta, principalmente a través de pescado azul, rico en VD, 0 de otros alimentos fortificados. La presencia de la enzima CYP27B1 y del receptor de la VD, distribuidos en las células y tejidos del organismo ${ }^{5}$ de modo casi universal y que alrededor del $3 \%$ del genoma humano está regulado por la hormona $1,25(\mathrm{OH}) 2 \mathrm{VD}^{6}$, le confieren a la VD importancia no sólo en la regulación del calcio y el metabolismo óseo, sino también en la inmunomodulación innata 0 adquirida, la regulación del crecimiento celular, la resistencia a la insulina, etc. En pacientes con enfermedad renal crónica, los niveles bajos de VD se relacionan con aumento de riesgo de diálisis y de mortalidad $^{1,5,7,8}$. Existe un consenso universal en que la medición en sangre de los niveles del metabolito $25(\mathrm{OH}) \mathrm{D}$ es el marcador del estatus corporal de VD, sea cual sea su procedencia ${ }^{7,9}$. Si bien no se sabe con certeza el nivel de normalidad, de los estudios realizados se sugiere que como mínimo deberían ser de $20 \mathrm{ng} /$ $\mathrm{ml}^{10}$, que el $37,3 \%$ de la población no los alcanzan y que incluso deberían ser más altos $5^{6,7,9,11}$.

El documento de posición sobre las necesidades y niveles óptimos de VD de la Sociedad Española de Inves- tigación Ósea y del Metabolismo Mineral (SEIOMM) y Sociedades afines sugiere que los niveles séricos de $25(\mathrm{OH}) \mathrm{D}$ entre 30 y $75 \mathrm{ng} / \mathrm{ml}$ parecen los más fisiológicos, y por tanto recomendables; que no se ha evidenciado toxicidad en pacientes con niveles de $25(\mathrm{OH}) \mathrm{D}$ por debajo de $100 \mathrm{ng} / \mathrm{ml}$ habiéndose propuesto que el umbral mínimo de toxicidad está por encima de los 150 $\mathrm{ng} / \mathrm{ml}(375 \mathrm{nmol} / \mathrm{l})^{11}$.

De los distintos estudios realizados en España, se extrae que del 41 al $87 \%$ de las personas tienen niveles inferiores a $20 \mathrm{ng} / \mathrm{ml}^{2}$ y que cuando se comparan las prevalencias de deficiencia de vitamina $D$ entre población sana y población con enfermedad renal crónica (ERC) en hemodiálisis (HD) la hipovitaminosis D se acentúa en esta última población (30-44\% vs $69-78 \%$ respectivamente) $)^{3,7}$.

Los niveles bajos de VD se han relacionado con localidades en latitudes superiores a $35^{\circ}$, mayor edad, ser mujer, menor exposición a la radiación UVB, filtros solares, escasa ingesta de VD, obesidad, diabetes mellitus, enfermedades intestinales que comprometan la absorción, enfermedades hepáticas que disminuyan la 25 -hidroxilación y la insuficiencia renal2,9,12. Además, en el enfermo renal, la disminuida fotobiosíntesis en la uremia, la proteinuria, la diálisis peritoneal, la reducción de la 1-alfa-hidroxilacion y de la reabsorcion tubular de 25(OH)VD, los inhibidores de la calcineurina y la disminución de la hidroxilación hepática de la VD asociada al hiperparatiroidismo secundario a la insuficiencia renal, son causas y factores de riesgo de niveles bajos de $\mathrm{VD}^{1,13}$.

\section{Objetivos}

Determinar la prevalencia de déficit e insuficiencia de VD y los factores de riesgo asociados en pacientes en HD.

\section{Material y Método}

Estudio descriptivo observacional de corte transversal, realizado en abril de 2017, coincidiendo con la determinación de los niveles sanguíneos de VD, mediante una entrevista oral sobre hábitos de adquisición de vitamina $\mathrm{D}$.

La población a estudio fueron los pacientes en tratamiento con HD en la clínica Fresenius Medical Care de Reus. Como criterios de inclusión se establecieron cualquier paciente, mayor de edad, que tuviese deter- 
minación analítica de la vitamina D (días 5-6 de abril de 2017) y quisieran responder a las preguntas. Se excluyeron los pacientes con dificultad idiomática y/o cognitiva, que no pudiesen colaborar en el estudio. Durante la fase de entrevistas hubo una mejoría del tiempo climático, soleado y con ascenso de las temperaturas, que invitaba a mayor exposición solar que la acontecida en meses previos. Consideramos que este hecho podría sesgar las respuestas por mayor exposición solar que la acontecida previa a la extracción de los niveles de VD obtenidos y paramos el estudio, quedando excluidos los pacientes no entrevistados.

Se diseñó un cuestionario ad hoc, que incluía las siguientes variables: edad, sexo, minutos de estancia al aire libre así como de exposición al sol, el uso de protección solar y factor de protección UV, superficie corporal expuesta, contaminación ambiental, ingesta de cualquier forma de alimentos enriquecidos con VD o alimentos ricos en VD como pescados grasos 0 aceite de hígado de bacalao. Dada la dificultad de cada paciente para cuantificar los gramos de VD de los alimentos ingeridos, se realizó una estimación aproximada de 50 o $100 \mathrm{~g}$. en función de la cantidad apreciada por él mismo. Los minutos de exposición reflejados son una estimación subjetiva del encuestado. Se documentó además la raza (asiática, blanca, negra) y el fototipo cutáneo (de 1 a 6 según la escala de Fitzpatrick) ${ }^{14}$. Como variables clínicas se recogieron enfermedades relacionadas, diabetes, malabsorción intestinal, insuficiencia hepática, tratamiento con estatinas, tratamiento con suplementación con VD, índice de masa corporal (IMC) y niveles séricos de VD.

Todos los pacientes incluidos en el estudio dieron su consentimiento informado por escrito para la inclusión y posible uso de sus datos médicos para fines científicos de forma anónima desde su historia clínica electrónica (EuClid) ${ }^{15}$.

Para el análisis de la población se definieron grupos de pacientes en función de sus niveles séricos de VD, clasificados como normales (niveles séricos de VD $\geq 30$ $\mathrm{ng} / \mathrm{ml}$ ); insuficiencia (niveles séricos de VD entre 15 y $30 \mathrm{ng} / \mathrm{ml}$ ) y déficit (niveles séricos de VD $<15 \mathrm{ng} / \mathrm{ml}$ ). Sin embargo, dada la controversia actual con estudios que consideran deficitarios niveles inferiores a $30 \mathrm{ng} /$ $\mathrm{ml}$, se decidió categorizar a los pacientes utilizando este punto de corte. De este modo, se plantearon dos análisis estadísticos al mismo tiempo comparando los cuatro grupos resultantes (Grupo Al:VD $<15 \mathrm{ng} / \mathrm{ml}$, Grupo A2: VD $\geq 15$ y $<19$ ng/ml, Grupo A3: VD $\geq 20$ y $<29 \mathrm{ng} / \mathrm{ml}$ y Grupo B:VD $\geq 30 \mathrm{ng} / \mathrm{ml}$ ) o tan solo dos de ellos (Grupo A total: VD $<30 \mathrm{ng} / \mathrm{ml}$ y Grupo B: VD $\geq 30 \mathrm{ng} / \mathrm{ml}$ ).

Todos los análisis estadísticos se realizaron con SPSS (23.0). Se contrastó la normalidad de las variables continuas con la prueba de Shapiro-Wilk. Para analizar estas variables se utilizaron los análisis estadísticos de Kruskal-Wallis o U de Mann-Whitney al comparar los cuatro o los dos grupos respectivamente. Por otro lado se utilizó el test Fisher para el análisis de las variables categóricas. Se consideraron como significativos aquellos valores de $p$ inferiores a 0.05 .

\section{Resultados}

Se analizaron 85 pacientes. En la tabla 1 se muestran la comparación de los resultados según niveles de VD. Solo el 5,88\% tuvieron niveles normales en un rango comprendido entre 33 y $48 \mathrm{ng} / \mathrm{ml}$ con un promedio de $38 \mathrm{ng} / \mathrm{ml}$. Resultaron pacientes más jóvenes, con menor porcentaje de mujeres, mayor exposición solar, sin uso de protectores, mayor ingesta de VD no farmacológica, mayor porcentaje de tratamiento con estatinas, pero también con mayor porcentaje de DM y obesidad-sobrepeso. Los mayores niveles de VD, $48 \mathrm{ng} / \mathrm{ml}$ los presentaba el único paciente asiático encuestado, que además de ser varón presentaba mayor ingesta de alimentos ricos en VD, normopeso, toma de rosuvastatina, no era diabético y no utilizaba protección.

El $94,14 \%$ de los pacientes presentaron niveles inferiores a $30 \mathrm{ng} / \mathrm{ml}$ (10,59\% insuficiencia y $83,53 \%$ déficit), con rango entre 4,10 y $20,27 \mathrm{ng} / \mathrm{ml}$ con un promedio de $9,64 \mathrm{ng} / \mathrm{ml}$. Estos pacientes resultaron ser más mayores, con mayor presencia de mujeres, menos expuestos al sol e incluso utilizan protección, menor ingesta de alimentos enriquecidos o ricos en $V D$, menos tratados con estatinas y presentan un porcentaje inferior de DM. Se encontraban en normopeso en mayor porcentaje, pese a contener los tres pacientes de la muestra con peso insuficiente. Los dos pacientes de raza negra tuvieron niveles séricos de VD $<10 \mathrm{ng} / \mathrm{ml}$.

\section{Discusión/Conclusiones}

En nuestra población, los niveles bajos de VD afectan al $94,14 \%$ de los pacientes en HD, siendo mayor de lo esperado por estar situada en una zona de turismo de sol. 
Tabla 1. Resultados según niveles de vitamina D (VD).

\begin{tabular}{|c|c|c|c|c|c|c|c|}
\hline Variable & Grupo Al & Grupo A2 & Grupo A3 & Grupo A total & Grupo B & pl & p2 \\
\hline Nivel sérico de VD (ng/ml) & $<15$ & $15-20$ & $20-30$ & $<30$ & $\geq 30$ & $\left|\begin{array}{c}4 \\
\text { grupos }\end{array}\right|$ & $\begin{array}{c}2 \\
\text { grupos }\end{array}$ \\
\hline Número de pacientes & 71 & 7 & 2 & 80 & 5 & & \\
\hline$\%$ de la muestra & $83,53 \%$ & $8,24 \%$ & $2,35 \%$ & $94,12 \%$ & $5,88 \%$ & & \\
\hline Edad (años) & $71,23(35-91)$ & $65(42-76)$ & $57(39-75)$ & $70(35-91)$ & $66(53-75)$ & 0,162 & 0,181 \\
\hline Sexo (\% mujeres) & $45,07 \%$ & $14,29 \%$ & $0,00 \%$ & $41,25 \%$ & $20,00 \%$ & 0,183 & 0,347 \\
\hline $\begin{array}{l}\text { Exposición en espacios abiertos } \\
\text { (minutos/ semana) }\end{array}$ & $649,01(0-4200)$ & $523(0-1680)$ & $427(14-840)$ & $632(0-4200)$ & $784(180-1428)$ & 0,725 & 0,316 \\
\hline $\begin{array}{l}\text { Exposición en espacios abiertos al sol } \\
\text { (minutos/ semana) }\end{array}$ & $269,86(0-2520)$ & $347(0-1260)$ & $315(0-630)$ & $278(0-2520)$ & 342 120-840) & 0,876 & 0,441 \\
\hline Uso de protector solar ( $\%$ SI) & $12,68 \%$ & $42,86 \%$ & $0,00 \%$ & $15,00 \%$ & $0,00 \%$ & 0,110 & 0,350 \\
\hline Factor protector solar usado (FPS) & $2,69(0-509)$ & $10,71(0-30)$ & 0 & $3,36(0-50)$ & 0 & 0,028 & 0,448 \\
\hline $\begin{array}{l}\text { Superficie corporal que recibe sol } \\
\text { (\% expuesta) }\end{array}$ & $11,03(0-64)$ & $12,86(0-21)$ & 6 & $11,06(0-64)$ & $13(9-18)$ & 0,350 & 0,434 \\
\hline $\begin{array}{l}\text { Ingesta alimentos enriquecidos con VD } \\
\text { ( } \% \text { ingesta positiva) }\end{array}$ & $12,68 \%$ & $14,29 \%$ & $0,00 \%$ & $12,50 \%$ & $20,00 \%$ & 0,911 & 0,628 \\
\hline Ingesta alimentos ricos en VD (gr/semana) & $103,59(0-300)$ & $85,71(0-200)$ & $75(50-100)$ & $101,31(0-300)$ & $130(50-200)$ & 0,625 & 0,299 \\
\hline Contaminación ambiental (\% expuestos) & 0 & 0 & 0 & 0 & 0 & - & - \\
\hline Malabsorción (\% SI) & 0 & 0 & 0 & 0 & 0 & - & - \\
\hline Tratamiento con estatinas ( $\%$ SI) & $35,71 \%$ & $57,14 \%$ & $0,00 \%$ & $36,71 \%$ & $60,00 \%$ & 0,322 & 0,298 \\
\hline Ingesta VD oral (\% SI) & $14,08 \%$ & $14,29 \%$ & $50,00 \%$ & $15,00 \%$ & $0,00 \%$ & 0,400 & 0,350 \\
\hline Fototipo (puntuación) & $3,1(1-6)$ & $3,29(3-4)$ & $3,5(3-4)$ & $3,14(1-6)$ & $3,6(3-4)$ & 0,321 & 0,119 \\
\hline Raza blanca (\% SI) & $84,15 \%$ & $8,54 \%$ & $2,44 \%$ & $95,12 \%$ & $4,88 \%$ & 0,011 & $<0,001$ \\
\hline Raza negra (\% SI) & $100,00 \%$ & $0,00 \%$ & $0,00 \%$ & $100,00 \%$ & $0,00 \%$ & & \\
\hline Raza asiática (\% SI) & $0,00 \%$ & $0,00 \%$ & $0,00 \%$ & $0,00 \%$ & $100,00 \%$ & & \\
\hline Diabetes (\% SI) & $36,62 \%$ & $28,57 \%$ & $0,00 \%$ & $35,00 \%$ & $60,00 \%$ & 0,470 & 0,260 \\
\hline Insuficiencia hepática (\% SI) & 0 & 0 & 0 & 0 & 0 & - & - \\
\hline IMC $\left(\mathrm{kg} / \mathrm{m}^{2}\right)$ & $25,79(12,66-38)$ & $24,99(21,56-30,41)$ & $29,94(24,88-35)$ & $25,8(12,7-38,4)$ & $29,1(24-35,5)$ & 0,305 & 0,142 \\
\hline Peso insuficiente IMC $<18,5$ (\% SI) & $100,00 \%$ & $0,00 \%$ & $0,00 \%$ & $100,00 \%$ & $0,00 \%$ & 0,528 & 0,601 \\
\hline Normopeso IMC 18,5-24,9 (\% SI) & $83,78 \%$ & $13,51 \%$ & $0,00 \%$ & $97,30 \%$ & $2,70 \%$ & & \\
\hline Sobrepeso IMC $25-29,9$ (\% SI) & $88,89 \%$ & $0,00 \%$ & $3,70 \%$ & $92,59 \%$ & $7,41 \%$ & & \\
\hline Obesidad IMC $=>30(\%$ SI $)$ & $72,22 \%$ & $11,11 \%$ & $5,56 \%$ & $88,89 \%$ & $11,11 \%$ & & \\
\hline Niveles VD (ng/ml) & $8,6(4,1-14,7)$ & $16,3(15-18,1)$ & $24,35(21,5-27,2)$ & $9,64(4,1-27,2)$ & $38(33-48)$ & $\mid<0,001$ & $<0,001$ \\
\hline
\end{tabular}

Grupo Al: niveles séricos de VD $<15 \mathrm{ng} / \mathrm{ml}$. Grupo A2: niveles séricos de VD $\geq 15 \mathrm{y}<20 \mathrm{ng} / \mathrm{ml}$. Grupo A3: niveles séricos de VD $\geq 20$ y $<30 \mathrm{ng} / \mathrm{ml}$. Grupo A total: niveles séricos de VD $<30 \mathrm{ng} / \mathrm{ml}$. Grupo B: niveles séricos de VD $\geq 30 \mathrm{ng} / \mathrm{ml}$. Para las variables cuantitativas se muestran los valores medios y el rango. $\mathrm{pl}$ : análisis según grupos Al, A2, A3 y B (Kruskal-Wallis/Test de Fisher). p2: análisis según grupos A total y B (U de Mann-Whitney/Test de Fisher).

Considerar el nivel normal como $\geq 30 \mathrm{ng} / \mathrm{ml}$, en vez de cifras inferiores, realizar la determinación de la VD al finalizar el invierno ${ }^{16}$, la localización y que solo el $15 \%$ de los encuestados tuviesen pautados suplementos de VD, favorecen cifras inferiores de VD a otros estudios $2,9,17,18,19$. No obstante prevalencias de $96 \%$ ya han sido descritas ${ }^{2,17}$.

Además de que por nuestra situación geográfica, Latitud: $41^{\circ} 09^{\prime} 22^{\prime \prime} \mathrm{N}$, en invierno la fotobiosíntesis de VD sea escasa, la falta de exposición solar (reclusión en la propia casa o residencias, o por la evitación del calor) la concienciación del daño que produce a nivel cutáneo con el consecuente rechazo del sol y protección mediante cremas, la ingesta pobre en VD (desaconsejada además por su elevado contenido en fósforo, restringido en nuestros pacientes) ${ }^{17}$, la edad y ser mujer parecen relacionarse con los niveles bajos. La toma de estatinas parece proteger la deficiencia de VD, concordando con el incremento de VD que puede aparecer con estatinas ${ }^{12,20}$. No se observó una relación negativa entre niveles de VD y obesidad o $\mathrm{DM}^{17}$. Tanto hay estudios con administración de VD muestran mejoría del control glucémico como que no, siendo difícil 
discernir el efecto entre VD y DM y no se encuentran pruebas suficientes para recomendarlas ${ }^{6,8}$.

Sólo el $14,12 \%$ de los pacientes tenían prescrito alguna suplementación de VD. Todos ellos tenían niveles $<30 \mathrm{ng} / \mathrm{ml}$, lo que puede explicarse por la inferior respuesta al tratamiento, la necesidad de dosis más altas en pacientes en $H^{19}$ y por falta de cumplimiento terapéutico que se favorece si la VD se administra en diálisis $^{21,22}$. No hemos podido observar que respondan mejor lo sujetos deficientes que insuficientes como se ha mencionado 6 .

El factor de protección solar utilizado se encontró como variable significativa entre grupos $(p=0.028)$. Su uso disminuye el aumento de VD tras la exposición solar' Para los pacientes con riesgo incrementado de cáncer de piel como los pacientes en diálisis y trasplantados se ha recomendado el uso de protectores solares y por ello también monitorización regular de los niveles de $\mathrm{VD}^{23}$.

Ninguna de las otras variables a estudio resultó tener una relación estadísticamente significativa, a excepción de la raza negra ( $p=0.011,2$ pacientes, ambos deficientes de VD). Las diferencias étnicas ya han sido descritas previamente ${ }^{9}$, aunque podrían ser atenuadas en localizaciones continuamente soleadas, sin diferencias estacionales, y aun así, no se aseguran niveles normales ${ }^{18}$.

Debemos tomar con precaución no encontrar otras variables significativas, debido al pequeño tamaño muestral de los grupos analizados y a que solo 5 de los pacientes $(5,88 \%$ de los encuestados) tuvieron niveles suficientes de VD. En un grupo de 58 pacientes, solo las restricciones dietéticas contribuyeron a la hipovitaminosis, ${ }^{17}$ en otro grupo, de 47 pacientes, fueron la exposición solar y la actividad al aire libre ${ }^{24}$.

La fortaleza de nuestro estudio se encuentra en que al tratarse de un solo centro y al realizarse las determinaciones de VD en el mismo momento, la población ha sido uniforme y el análisis de los datos congruentes.

Dado que los niveles bajos de VD en la población con enfermedad renal crónica y en diálisis ha sido asociada con hiperparatiroidismo secundario y marcadores de alto turnover óseo, baja densidad ósea, debilidad muscular y riesgo de caídas, síndrome metabólico, obesidad y resistencia a la insulina, hipertrofia ventri- cular izquierda y ateroesclerosis, calcificación vascular y rigidez arterial, deterioro cognitivo, progresión de la enfermedad renal y mortalidad, y que la suplementación con VD puede mejorar los niveles de PTH, la proteinuria, los niveles de $1,25(\mathrm{OH}) \mathrm{D}$, los marcadores endoteliales cardiovasculares y los parámetros inflamatorios ${ }^{1,9,13}$, y teniendo en cuenta que puede ser administrada con seguridad8,19,21,25,26, debería ampliarse la suplementación en estos pacientes.

A la vista de estos resultados encontramos que el $94,14 \%$ de la población en HD en Reus presenta niveles bajos de VD, finalizado el invierno. Los datos estudiados muestran la necesidad de la suplementación con VD farmacológica si queremos normalizar los niveles de VD tal como sugieren las recomendaciones ${ }^{27,28}$ y proteger a nuestros pacientes de los efectos adversos relacionados con niveles séricos de VD bajos.

\section{El autor declara que no hay conflicto de interés.}

Recibido: 21 julio 2017

Revisado: 15 agosto 2017

Modificado: 25 septiembre 2017

Aceptado: 15 diciembre 2017

\section{Bibliografía}

1. Dusso AS. Kidney disease and vitamin D levels: 25-hydroxyvitamin D, 1,25-dihydroxyvitamin D, and VDR activation. Kidney Int. 2011;1(4): 136141.

2. Marinelli $A$, Pistolesi $V$, Rossi $V$, Battista M,Buono A, Della Grotta F et al. Severe $25-0 H$ vitamin D deficiency in patients on chronic hemodialysis.G Ital Nefrol. 2014;31(5): 1-8.

3. Hilger J, Friedel A, Herr R, Rausch $T$, Roos $F$, Wahl DA et al. A systematic review of vitamin $D$ status in populations worldwide. Br J Nutr. 2014; 111(1): 23-45.

4. Navarro C, Quesada JM. Deficiencia de vitamina D en España. ¿Realidad o mito? Rev Osteoporos Metab Miner. 2014; 6 (Supl 1):85-10. 
5. González ML. Vitamina D en la enfermedad renal crónica. Nefrología al día 2012; 7:0 | doi: 10.3265/ Nefrologia.2010.publ.ed80.chapter2930.

6. Navarro C, Quesada JM. Vitamina D, determinante de la salud osea y extraósea; importancia de su suplementación en la leche y derivados. Nutr Hosp. 2015;3 (supl 2): 18-25.

7. Ravani $P$, Malberti F, Tripepi G, Pecchini P, Cutrupi $S$, Pizzini $P$, et al. Vitamin D levels and patient outcome in chronic kidney disease. Kidney Int. 2009; 75(1): 88-95.

8. Parikh C, Gutgarts V, Eisenberg E, Melamed ML. Vitamin $D$ and clinical outcomes in dialysis. Semin Dial. 2015; 28(6): 604-609.

9. Wolpowitz D, Gilchrest BA. The vitamin D questions: how much do you need and how should you get it? J Am Acad Dermatol. 2006;54(2):301-17.

10. Ross AC, Manson JE, Abrams SA, Aloia JF, Brannon PM, Clinton SK et al . The 2011 report on dietary reference intakes for calcium and vitamin $D$ from the Institute of Medicine: What clinicians need to know? J Clin Endocrinol Metab. 2011; 96(1): 53-58.

11. Gómez de Tejada MJ, Sosa M, Del Pino J, Jodar E, Quesada JM, Cancelo MJ. Documento de posición sobre las necesidades y niveles óptimos de vitamina D. Rev Osteoporos Metab Miner. 2011; 3(1): 53-64.

12. Moyad MA. Vitamin D: A rapid review. Dermatol Nurs. 2009;21(1): 25-30, 55.

13. Jean G, Souberbielle JC, Chazot C. Vitamin D in chronic kidney disease and dialysis patients. Nutrients $2017 ; 9(4): 328$.

14. ANEDIDIC. Procedimiento de actuación ante la exposición al sol. Enferm Dermatol 2012; 15: 48-49.

15. Pérez-García R, Palomares I, Merello JI, Aljama $\mathrm{P}$, Bustamante J, Luño J et al; (ORD group). Epidemiological study of 7316 patients on haemodialysis treated in FME clinics in Spain, using data from the EuCliD database: results from years 2009-2010. Nefrología. 2012; 32:743-753.

16. Fernández A, Donnay S, Beamud M. Seasonal variation in vitamin $D$ levels in patients attending in
Basic Helathcare Center. Rev Osteoporos Metab Miner 2012; 4(2): 63-68.

17. Krassilnikova M, Ostrow $K$, Bader $A$, Heeger $P$, Mehrotra A. Low dietary intake of vitamin $D$ and vitamin $\mathrm{D}$ deficiency in hemodialysis patients. $\mathrm{J}$ Nephrol Ther. 2014; 4(3): 166.

18. Foucan L, Ducros J, Merault H. Vitamin D status in dark-skinned patients undergoing hemodialysis in a continually sunny country. J Nephrol. 2012; 25 (6): 983-8.

19. Ojeda R, Esquivias E, Carmona A, García V, Berdud I, Martí A et al. La corrección del déficit de 25-0H-vitamina D mejora el control del hiperparatiroidismo secundario y el estado inflamatorio de pacientes estables en hemodiálisis. Nefrologia. 2018; 38(1):41-47.

20. Pérez-Castrillón JL, Abad L, Vega G, Sanz-Cantalapiedra A, García-Porrero M, Pinacho $F$ et al. Effect of atorvastatin on bone mineral density in patients with acute coronary syndrome. Eur Rev Med Pharmacol Sci. 2008;12(2):83-8. Abstract.

21. Subih HS, Behrens J, Burt B, Clement L, Pannell $R$, Macha $L$ et al. 25 hydroxy vitamin $D$ is higher when a renal multivitamin is given with cholecalciferol at hemodialysis. Asia Pac J Clin Nutr. 2016; 25(4):754-759.

22. Sánchez D, Pereira M, González E, Pérez MV. La enfermería pieza clave en el tratamiento del déficit de vitamina D. Enferm Nefrol. 2013; 16(supl 1): 83-84.

23. Ulrich C, Deegen A, Patel MJ, Stockfleth E. Sunscreens in organ transplant patients. Nephrol Dial Transplant. 2008; 3(6):1805-1808.

24. Lee YJ, Oh IH, Baek HJ, Lee CH, Lee SS. Effects of sun exposure and dietary vitamin $D$ intake on serum 25-hydroxyvitamin $D$ status in hemodialysis patients. Nutr Res Pract. 2015; 9(2):158-64

25. Kalantar-Zadeh K, Fouque D. Nutritional management of chronic kidney disease. N Engl J Med. 2017;377(18):1765-1776.

26. Ramirez B, De Jesús $M$, Pulido $M$, Roncal D, Rodríguez $\mathrm{P}$. Hiperparatiroidismo secundario. Una 
puesta al día. Rev Soc Esp Enferm Nefrol 2001; 4(4): 56-61.

27. Kidney Disease: Improving Global Outcomes (KDIGO) CKD-MBD Work Group. KDIGO clinical practice guideline for the diagnosis, evaluation, prevention, and treatment of chronic kidney disease-mineral and bone disorder (CKD-MBD). Kidney Int. 2009; 76 (Suppl 113): S1-S130.

28. Vicente J, Bover J, Cannata J, Lorenzo V, Martín de Francisco AL, Martínez I et al. Recomendaciones de la Sociedad Española de Nefrología para el manejo de las alteraciones del metabolismo óseo-mineral en los pacientes con enfermedad renal crónica (S.E.N.-M.M.). Nefrología. 2011; 31(1); 3-32.

Este artículo se distribuye bajo una Licencia Creative Commons Atribución-NoComercial 4.0 Internacional. https://creativecommons.org/licenses/by-nc/4.0/

\section{Open Access (C) ()}

